\title{
Sensoren für die zukünftige Wasserstoffwirtschaft
}

\author{
Jens Zosel ${ }^{1}$, Michael Mertig ${ }^{1,2}$ \\ ${ }^{1}$ Kurt-Schwabe-Institut für Mess- und Sensortechnik Meinsberg e.V. (KSI), \\ Kurt-Schwabe-Straße 4, 04736 Waldheim, Deutschland \\ ${ }^{7}$ Professur für Physikalische Chemie, Mess- und Sensortechnik, Technische Universität Dresden, \\ 01062 Dresden, Deutschland \\ Kontakt: jens.zosel@ksi-meinsberg.de
}

\section{Einleitung}

Als chemischer Grund-, Prozess- und Hilfsstoff wird Wasserstoff $\left(\mathrm{H}_{2}\right)$ bereits seit vielen Jahrzehnten in einer Vielzahl von Industriezweigen verwendet [1]. Geschultes Personal und eine ausgereifte Sicherheitstechnik gewährleisten hier den sicheren Umgang mit $\mathrm{H}_{2}$ [2]. Jedoch wird dieses Gas in immer mehr Anwendungen als sauberer Energieträger aus erneuerbaren Quellen in Erwägung gezogen und zunehmend eingesetzt. Beispiele dafür sind die Entwicklung von Brennstoffzellenfahrzeugen aller Leistungsklassen [3], der Aufbau von Hochleistungs-Elektrolysesystemen für die Umsetzung überschüssiger Elektroenergie aus Wind-, Wasser- und Solarkraftwerken (power-to-gas) [4, 5] und der dezentrale Einsatz von $\mathrm{H}_{2}$ als Energieträger auch im Heimbereich [6]. Diese Applikationen führen neben einer Dezentralisierung der $\mathrm{H}_{2}$-Versorgungskette von der Herstellung über den Transport und die Zwischenspeicherung bis hin zu den Endnutzern auch zum zunehmenden Umgang mit $\mathrm{H}_{2}$ durch ungeschultes Personal.

Auf der anderen Seite nimmt $\mathrm{H}_{2}$ wegen mehrerer Besonderheiten als Gefahrstoff eine Sonderstellung unter den bislang verwendeten fluiden Energieträgern ein. Die wohl wichtigste sicherheitsrelevante Eigenschaft betrifft die äußerst geringe Zündenergie von $16 \mu \mathrm{J}$, die mit einem vergleichsweise weiten Zündbereich der $\mathrm{H}_{2}$-Volumenkonzentration an Luft zwischen $4 \%$ und $75 \%$ sowie einer sehr geringen Dichte verknüpft ist. Diese Eigenschaften führten dazu, dass bei der Mehrzahl der bislang eingehend untersuchten Unfälle mit gezündeten $\mathrm{H}_{2}$-Luft-Gemischen die Zündquelle nicht aufgeklärt werden konnte [7]. Heute werden deshalb in zunehmenden Maß Selbstentzündungsmechanismen austretender $\mathrm{H}_{2}$-Hochdruck-Jets untersucht [8]. Zudem ist $\mathrm{H}_{2}$ gemäß Abb. 1 [9] in einem sehr weiten Volumenstrombereich zündfähig. Ein weiteres Risiko im Umgang mit $\mathrm{H}_{2}$ besteht darin, dass Wasserstoffflammen für das menschliche Auge weitgehend unsichtbar sind und so bereits häufiger vor ihrer Entdeckung signifikante Sekundärschäden verursacht haben. Aus diesen vielfältigen Risiken resultiert neben der Notwendigkeit einer angepassten Auslegung von $\mathrm{H}_{2}$-enthaltenden Anlagen auch ein kontinuierliches Sicherheitsmonitoring der Infrastrukturen der neu entstehenden $\mathrm{H}_{2}$-Wirtschaft. Dafür müssen hochsensitive und hochselektive $\mathrm{H}_{2}$-Sensoren mit unteren Messgrenzen $<10$ Vol.-ppm vorhanden sein, deren Signale intelligent verarbeitet und automatisiert mit aktiven Sicherheitskomponenten verknüpft werden müssen [10].

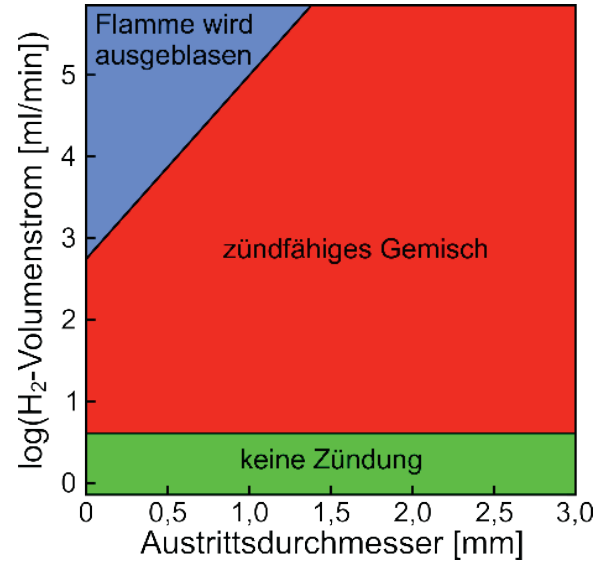

Abb. 1: Bereich der Entstehung zündfähiger Gemische bei Austritt von $\mathrm{H}_{2}$ an Luft (nach [9]).

Neben der Leckageüberwachung werden im zunehmendem Maß auch Sensoren für die Überwachung der Konzentration und der Reinheit von $\mathrm{H}_{2}$ in den Infrastrukturen für dessen Herstellung, Verteilung, Lagerung und Verarbeitung benötigt. Obwohl für diese neuen Anwendungsfelder bereits heute viele verschiedene $\mathrm{H}_{2}$-Sensoren kommerziell verfügbar sind [11], bleiben dennoch immer noch wichtige Forderungen der Anwenderseite unerfüllt, die neben Selektivität, Zuverlässigkeit und Kosten auch erweiterte Messbereiche und äußere Bedingungen wie hohe Drücke und Gasfeuchten betreffen [12]. Deshalb wurden im abgeschlossenen Verbundvorhaben „Prozess- und Sicherheitssensorik für das Wasserstoff-Qualitätsmanagement (HyProS)" die in Abb. 2 dargestellten $\mathrm{H}_{2}$-Sensoren entwickelt, die im vorliegenden Beitrag näher vorgestellt werden.

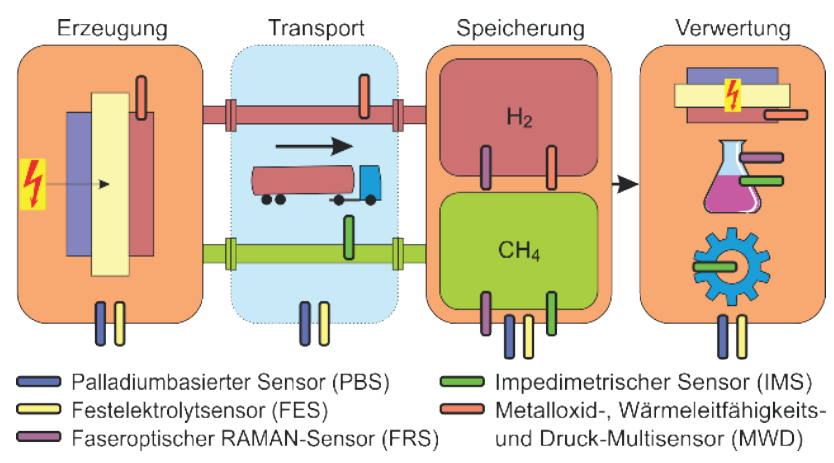

Abb. 2: Schematische Darstellung der Applikation von Sensoren und Messsystemen, die im Verbundvorhaben HyProS entwickelt wurden. 


\section{Methoden und Materialien}

Für die Leckageüberwachung an wasserstoffverarbeitenden Anlagen wurden im Vorhaben hochminiaturisierte palladiumbasierte Sensoren (PBS) entwickelt, die im explosionsgeschützten Bereich einsetzbar sind [13]. Der Messbereich dieser Sensoren erstreckt sich von etwa 100 Vol.-ppm bis 100 Vol.-\%. Um diesen bis zu Spurenkonzentrationen ab 0,5 Vol.-ppm zu erweitern, wurden diese Sensoren mit coulometrisch arbeitenden Festelektrolytsensoren (FES) kombiniert [14], die zur Selektivitätsverbesserung eine chromatographische Vortrennung des $\mathrm{H}_{2}$-haltigen Messgases aufweisen.

Die Überwachung von Wasserstoff in Erdgasnetzen im Konzentrationsbereich bis 20 Vol.- $\%$ stellt eine weitere neue Applikation in der modernen Wasserstoffwirtschaft dar, da auf diese Weise beispielsweise der in OffshoreWindparks erzeugte Wasserstoff zu den Bedarfsträgern in den Industriegebieten ohne größere Investitionen transportiert werden kann. Dafür wurden im Vorhaben katalytischimpedimetrische $\mathrm{H}_{2}$-Sensoren (IMS) [15] sowie faseroptische Raman-Sensoren (FRS) [16] entwickelt und erprobt. Diese beiden Entwicklungsrichtungen wurden durch ein diversitär-redundantes $\mathrm{H}_{2}$-Multisensorsystem (MWD), komplettiert, das aus einem selektivem Metalloxid(MOX)-Halbleitergassensor, einem miniaturisierten Wärmeleitfähigkeitsdetektor und einem $\mathrm{H}_{2}$-stabilen Drucksensor besteht [17]. Die Signale dieser Einzelsensoren werden dabei durch eine intelligente Signalverarbeitung verknüpft, um beispielsweise in $\mathrm{H}_{2}$-Druckspeichern bis 100 bar die Qualität des eingelagerten $\mathrm{H}_{2}$ lückenlos überwachen zu können.

Themenübergreifend waren im Vorhaben weiterhin Querschnittsaufgaben vorgesehen, in denen Fragestellungen zur Materialauswahl für die Sensoren, zu deren Zuverlässigkeit und zu Alterungsvorgängen unter rauen Umgebungsbedingungen bearbeitet wurden. Weiterhin wurden im Rahmen dieser Querschnittsaufgaben Simulationsrechnungen zur Optimierung von Sensorlayouts, zu Bauteildeformationen und zu den Einsatzbedingungen der Sensoren in den o.g. Applikationen des erweiterten Projektkonsortiums im HYPOS-Verein durchgeführt [18].

\section{Beschreibung der Sensoren und Messprinzipien}

A) IMS

Der gemeinsam von der Hochschule für Technik und Wirtschaft und Kultur, Leipzig (HTWK), dem Leibniz-Institut für Katalyse e.V., Rostock, der Sciospec GmbH, Wurzen, und der Union Instruments $\mathrm{GmbH}$, Karlsruhe, entwickelte IMS beruht auf der Erfassung des Spillover-H2. Das in Abb. 3 dargestellte Prinzipschema dieses Sensor zeigt, dass die in der Gasphase vor dem Sensor vorhandenen Wasserstoffmoleküle an einer mit Platin-Keimen versehenen Oberfläche aus einem platinhaltigen $\mathrm{H}_{2}$-Akzeptor adsorbieren und dort in einzelne angeregte $\mathrm{H}$-Atome dissoziieren. Diese Atome diffundieren an der Sensoroberfläche in einem zweiten Schritt auf einen für die $\mathrm{H}_{2}$-Dissoziation nicht katalytisch aktiven Bereich (Spillover) und binden dort unter Abgabe eines Elektrons an oberflächliche Sauerstoffatome unter
Bildung von $\mathrm{OH}-M o l e k u ̈ l e n$. Die Oberflächenleitfähigkeit des $\mathrm{H}_{2}$-Akzeptors wird durch diese Moleküle modifiziert und kann mit Messungen der komplexen Wechselstromimpedanz an Interdigitalelektroden auf der Oberfläche des Akzeptors ausgelesen werden. $\mathrm{Da} \mathrm{H}_{2}$ als einziges Molekül im Gasstrom schnell und reversibel mit dieser speziell gestalteten Oberfläche wechselwirkt, ist das Sensorsignal hochselektiv und weist auch in komplexen Gasgemischen mit vielen verschiedenen Einzelkomponenten wenig Querempfindlichkeiten auf. Für die Signalbildung muss bei diesem Sensor kein Sauerstoff im Messgas vorhanden sein.

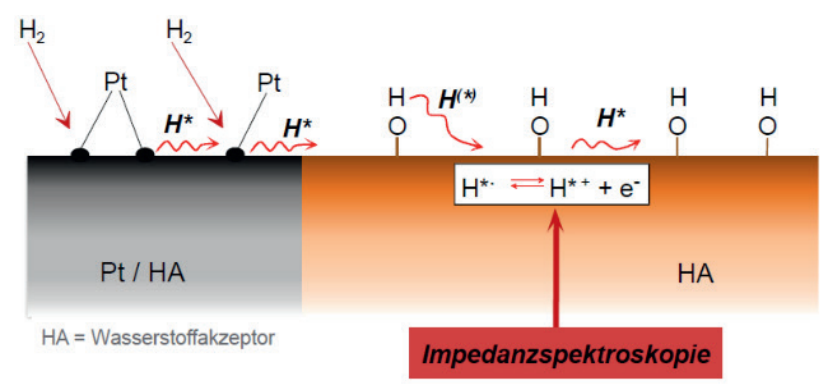

Abb. 3: Funktionsschema eines impedimetrischen Sensors zur Messung des Spillover-Wasserstoffs.

B) FRS

Das in Abb. 4 aufgeführte Funktionsschema der selektiven Gasdetektion mittels Raman-Streuung wurde im Teilvorhaben für die Entwicklung und Erprobung eines hochselektiven faseroptischen Sensors am Leibniz-Institut für Photonische Technologien e.V., Jena, genutzt. Bei diesem Sensor wird ein spezifisch angepasster Laserstrahl in eine speziell entwickelte Hohlkernfaser eingekoppelt, durch die das Messgas strömt. Auf dem Weg des Lichts durch die Faser kommt es in Abhängigkeit von der $\mathrm{H}_{2}$-Konzentration zu zahlreichen inelastischen Streuvorgängen an $\mathrm{H}_{2}$-Molekülen. Dadurch treten im Streulichtspektrum neben der Laser-Wellenlänge $v_{i}$ Seitenbänder bei den Frequenzen $v_{i} \pm$ $v_{\text {vib }}$ auf, die durch den Raman-Effekt erster Ordnung verursacht werden [19].

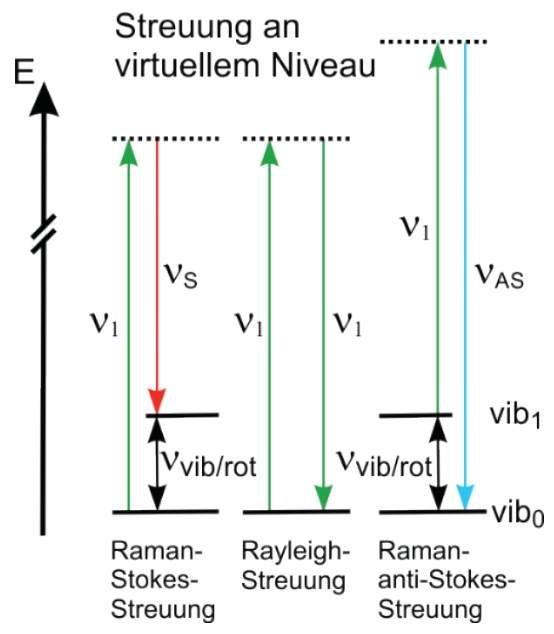

Abb. 4: Prinzipschema der selektiven $\mathrm{H}_{2}$-Messung mittels Raman-Streuung. 
Die Intensität dieser Seitenbänder hängt von der Konzentration der korrespondierenden $\mathrm{H}_{2}$-Moleküle ab. Da die Schwingungsfrequenz vvib von $\mathrm{H}_{2}$ selektiv für dieses Molekül ist und sich von Frequenzen anderer Gasmoleküle deutlich unterscheidet, kann dieser Analyt mittels Spektrometer und hochempfindlichem Detektor mit hoher Selektivität bis herunter zu Konzentrationen im oberen ppm-Bereich erfasst werden.

\section{C) MWD}

Von der Umweltsensortechnik UST GmbH, Geschwenda, und der CiS Forschungsinstitut für Mikrosensorik $\mathrm{GmbH}$, Erfurt, wurde das in Abb. 5 gezeigte Konzept des diversitärredundanten $\mathrm{H}_{2}$-Multisensorsystems entwickelt und erprobt. Bei diesem Ansatz werden die Signale dreier Einzelsensoren miteinander intelligent verknüpft, die jeweils ganz unterschiedliche Informationen aus dem Messgas liefern. Während mit dem Wärmeleitfähigkeitsdetektor vor allem die Konzentration des vorhandenen Wasserstoffs charakterisiert werden kann, liefert der parallel ausgelesene Multielektroden-MOX-Sensor Informationen zu möglichen Verunreinigungen, die beispielsweise in Kavernenspeichern oder Elektrolyseuren auftreten können. Der Drucksensor dient einerseits zum Abgleich der Signale der beiden anderen Sensoren und wird andererseits auch für die Druckmessung selbst an der Messstelle benötigt.

D) PBS

Das Prinzip des palladiumbasierten $\mathrm{H}_{2}$-Sensors wurde von der Materion $\mathrm{GmbH}$, Wismar, entwickelt und gemeinsam mit dem KSI Meinsberg und der Analytical Control Instruments $\mathrm{GmbH}(\mathrm{ACl})$, Berlin, zu einem industrietauglichen Sensor für den Einsatz in explosionsgeschützten Bereichen komplettiert.

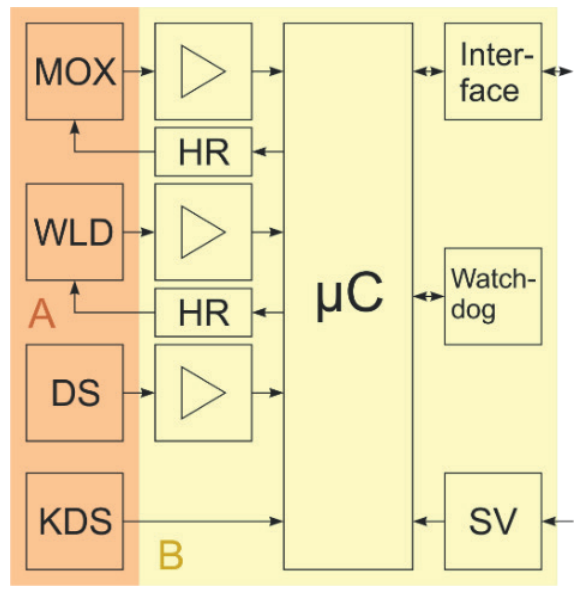

Abb. 5: Funktionsschema des diversitär-redundanten $\mathrm{H}_{2-}$ Multisensorsystems für die Überwachung von Großspeichern und Elektrolyseuren; MOX $=$ Metalloxid-Gassensor,

WLD $=$ Wärmeleitfähigkeitsdetektor, DS = Drucksensor, KDS $=$ Kalibrierdatenspeicher, HR = Heizungsregelung, $\mu \mathrm{C}=$ Mikrocontroller, SV $=$ Stromversorgung, $\mathrm{A}=$ austauschbarer intelligenter Sensorkopf, $\mathrm{B}=$ Datenaufnahme und -verarbeitung.
Für diesen Sensor wurde die seit längerem bestehende Erkenntnis genutzt, dass sich Eigenschaften wie Farbe, Leitfähigkeit oder mechanische Spannung von Palladium $(\mathrm{Pd})$ ändern, sobald sich $\mathrm{H}_{2}$ in das Metall-Gefüge einlagert. Dabei ermöglicht Pd die Lösung höherer $\mathrm{H}_{2}$-Mengen und ändert dabei seine optischen, elektrischen und mechanischen Eigenschaften erheblich. Bei den hier entwickelten Sensoren wurde der reversible Effekt der Volumenvergrößerung bei $\mathrm{H}_{2}$-Einlagerung in $\mathrm{Pd}$ sowie in $\mathrm{Pd}$-haltige Legierungen genutzt. Die Wasserstoffkonzentration und die damit verbundene Ausdehnung einer Pd-haltigen Schicht wurde mit dem in Abb. 6 gezeigten mikroelektromechanischen System (MEMS) durch Dehnungsmessung erfasst.

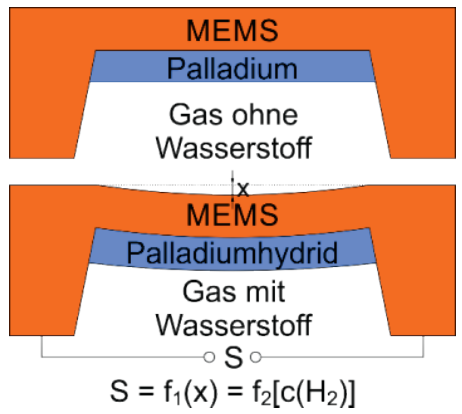

Abb. 6: Prinzipschema der selektiven $\mathrm{H}_{2}$-Messung mittels Dehnungsmessung an $\mathrm{H}_{2}$ einlagernden palladiumhaltigen Schichten.

Dazu wird das Signal S eines PBS als Funktion der Durchbiegung $x$ ermittelt, die ihrerseits von der Menge des eingelagerten Wasserstoffs und somit vom $\mathrm{H}_{2}$-Partialdruck in der Gasphase abhängt. Somit korreliert das Signal S mit der $\mathrm{H}_{2}$-Konzentration in der Gasphase. Durch die Aufbringung sehr dünner Pd-haltiger Schichten werden vergleichsweise kurze Ansprechzeiten von wenigen Sekunden erreicht.

\section{E) FES}

Das gemeinsam von $\mathrm{KSI}$ Meinsberg und $\mathrm{ACl}$ entwickelte Konzept eines industrietauglichen $\mathrm{H}_{2}$-Messsystems auf der Basis eines coulometrischen Festelektrolytsensors wurde für die verteilte $\mathrm{H}_{2}$-Leckageüberwachung geschaffen. Die $\mathrm{H}_{2}$-Selektivität dieses Messsystems, das mit einem nichtselektiven coulometrischen Festelektrolytsensor auf der Basis von stabilisiertem Zirconiumdioxid ausgerüstet ist, wurde wie in Abb. 7 gezeigt durch eine chromatographische Vortrennung des Messgasgemisches gewährleistet. Durch diese Vortrennung wird zudem sichergestellt, dass sich im heißen Festelektrolyt-Gassensor keine endzündbaren Messgasgemische bilden können. Die Trennung erfolgte in chromatographischen Säulen. Der Festelektrolytsensor wird dann als schnell ansprechender coulometrischer Detektor eingesetzt, indem durch konstante Polarisation der Messelektrode gegenüber einer Pt-Luft-Referenzelektrode ein gleichbleibender Sauerstoffpartialdruck im Bereich 0,01 - 0,1 Pa eingestellt wird. Ein einlaufender $\mathrm{H}_{2}$-Peak wird in einem sauerstofffreien Trägergas durch einen positiven Faraday-Strom angezeigt, während reduzierbare Analyte wie $\mathrm{O}_{2}$ einen negativen Peak hervorrufen. Die Peakfläche ist ein absolutes Maß für die injizierte Analytmenge. 


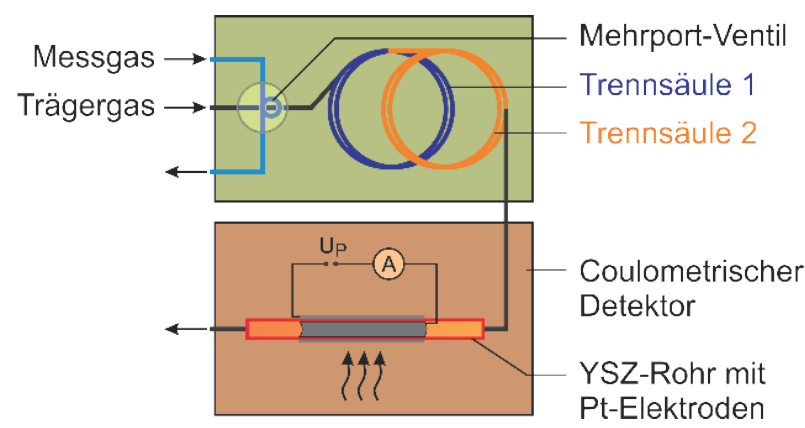

Abb. 7: Schema des Festelektrolytsensors mit chromatographischer Vortrennung zur selektiven Messung von $\mathrm{H}_{2}$-Spurenkonzentrationen im ppb-Bereich.

Die Vorteile dieses schnellen Detektors bestehen in seinem breiten Messbereich über etwa 6 Zehnerpotenzen und seiner hohen Langzeitstabilität, die kalibrierfreie Messungen von vielen Monaten ermöglicht. Ein weiterer Vorteil dieses Messsystems besteht in der inhärenten Möglichkeit, durch die chromatographische Trennung parallel $\mathrm{zu} \mathrm{H}_{2}$ auch weitere Analyte bestimmen zu können, wodurch sich auch potentielle Applikationen bei der Qualitätsüberwachung ergeben.

\section{Ergebnisse}

\section{A) IMS}

Der in Abb. 8 dargestellte Versuchsstand zur Erprobung von IMS wurde an der HTWK aufgebaut. Für die impedimetrische Messung und die erweiterte Signalanalyse wurden von Sciospec miniaturisierte Multisensor-Impedanzspektrometer neu entwickelt und erfolgreich eingesetzt. Der Prüfstand ermöglicht den flexiblen Einsatz verschiedener Gasmischungen zur Nachbildung der Einsatzbedingungen in Erdgasnetzen. Die in Abb. 9 als Beispiel aufgeführten Bodeplots belegen, dass die gewählten Sensorbeschichtungen den Spillover-Effekt zeigen und bereits ab $\mathrm{H}_{2}$-Konzentrationen von $1 \mathrm{Vol}$.- \% zu deutlichen Änderungen des Betrages und der Phase der komplexen Wechselstromimpedanz führen. Mit zunehmender $\mathrm{H}_{2}$-Konzentration ändert sich der Betrag der komplexen Impedanz vor allem bei niedrigen Frequenzen signifikant. Die Sensitivität der Phasenverschiebung auf $\mathrm{H}_{2}$ ist bei Frequenzen um $1 \mathrm{kHz}$ besonders groß.

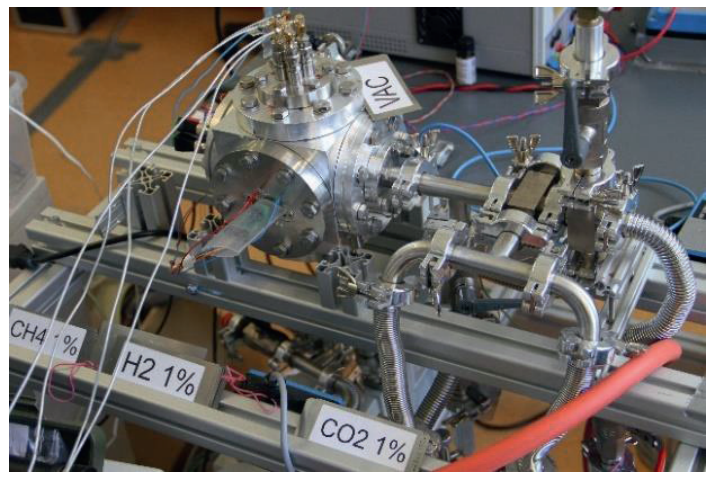

Abb. 8: Versuchsaufbau zur Charakterisierung impedimetrischer $\mathrm{H}_{2}$-Sensoren.

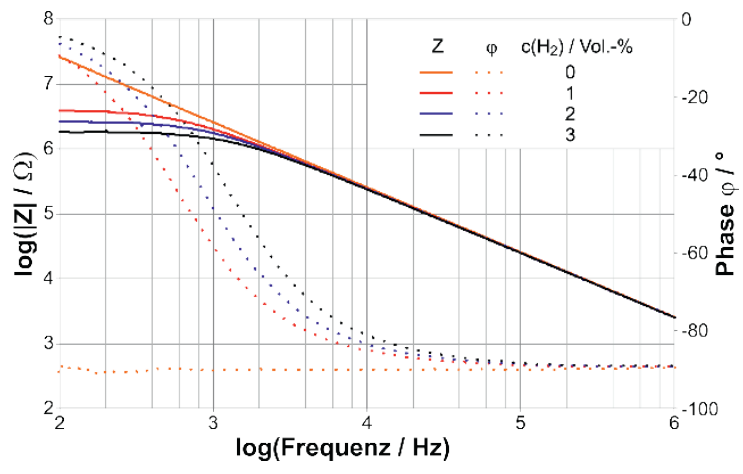

Abb. 9: Impedanz-Bodeplots an einem IMS bei verschiedenen $\mathrm{H}_{2}$-Konzentrationen.

\section{B) MWS}

Vom diversitär-redundanten $\mathrm{H}_{2}$-Multisensor ist in Abb. 10 der Signalverlauf eines miniaturisierten WLD-Sensors als Beispiel dargestellt. Die Kurve belegt dessen hohe Sensitivität und Ansprechgeschwindigkeit. In Kombination mit den Signalen der ebenfalls integrierten Metalloxid- und Drucksensoren lassen sich neben der Wärmeleitfähigkeit noch weitere für die Beurteilung der Wasserstoffqualität wichtige Informationen aus dem Messgas gewinnen. Weitere Resultate zum diesem praxisreifen Sensor sind in [17] publiziert worden.

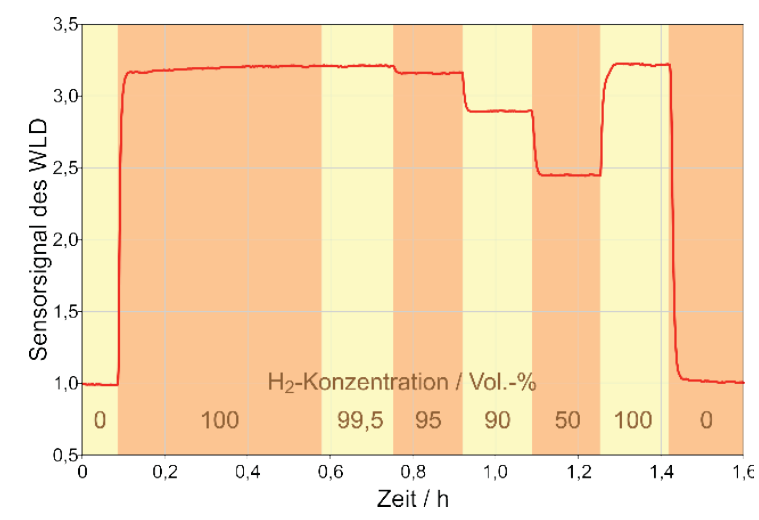

Abb. 10: Signalverlauf eines gemäß Abb. 5 im MWD integrierten Wärmeleitfähigkeitsdetektors (WLD) bei verschiedenen Konzentrationen $\mathrm{c}\left(\mathrm{H}_{2}\right)$.

C) PBS

In Abb. 11 ist eine typische Signalkurve eines PBS in Gasen mit verschiedenen $\mathrm{H}_{2}$-Konzentrationen dargestellt. Das Ergebnis belegt kurze Ansprechzeiten unter $30 \mathrm{~s}$, die weitgehend unabhängig von der Richtung des Konzentrationswechsels sind. Weiterhin zeigt das untersuchte Versuchsmuster nach einer Warmlaufphase von einem Beladungszyklus eine ausgezeichnete Wiederholgenauigkeit des Spannungssignals zwischen den einzelnen Zyklen, das logarithmisch von der $\mathrm{H}_{2}$-Konzentration abhängt [13]. Bei konstanter Konzentration treten nach Abschluss des Einstellvorgangs keine Signaldriften auf. Durch das Fehlen elektrischer Energieeinträge in den Sensor und die optische Signalweiterleitung ist der Sensoreinsatz in explosionsgeschützten Bereichen problemlos möglich. 


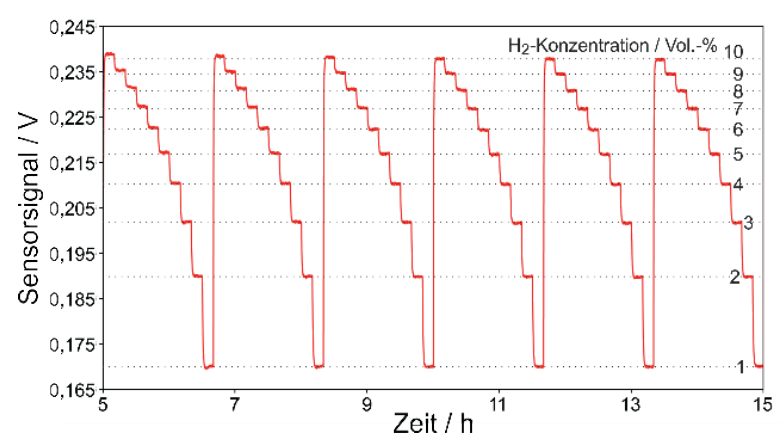

Abb. 11: Signalverlauf eines palladiumbasierten Sensors bei wechselnden $\mathrm{H}_{2}$-Konzentrationen.

\section{D) FES}

Der im Vorhaben entwickelte Prototyp eines FES mit chromatographischer Vortrennung wurde in ein 19-Zoll-Gehäuse integriert, wie in Abb. 12 gezeigt ist. An dem dort verwendeten Festelektrolyt-Detektor wurden die in Abb. 13 dargestellten Ausschnitte von Chromatogrammen aufgezeichnet. Die Kurven belegen, dass $\mathrm{H}_{2}$ in den chromatographischen Säulen ausreichend vom darauffolgenden $\mathrm{O}_{2-}$ Peak getrennt und als positiver Peak vollständig aufgezeichnet wird. Die langzeitstabile Funktion des Detektors auf der Basis des Faraday-Gesetzes, die bei vollständigem Umsatz der zu messenden Gaskomponenten im Detektor gegeben ist, ermöglicht einen kalibrierfreien Betrieb [14].

E) Materialauswahl und Zuverlässigkeit

Neben den Arbeiten an den Sensorprinzipien und entsprechenden Versuchsmustern erfolgten im Vorhaben am Fraunhofer-Institut für Mikrostruktur von Werkstoffen und Systemen IMWS, Halle, und an der Technischen Universität Bergakademie Freiberg Untersuchungen zur Materialauswahl für die Sensoren und zur Zuverlässigkeit von Strukturelementen. Damit konnten Alterungsvorgänge aufgeklärt werden, die vor allem unter den im Feldbetrieb teilweise auftretenden rauen Umgebungsbedingungen die Sensorstandzeit begrenzen können. Ein Beispiel für diese Untersuchungen ist in Abb. 14 illustriert.

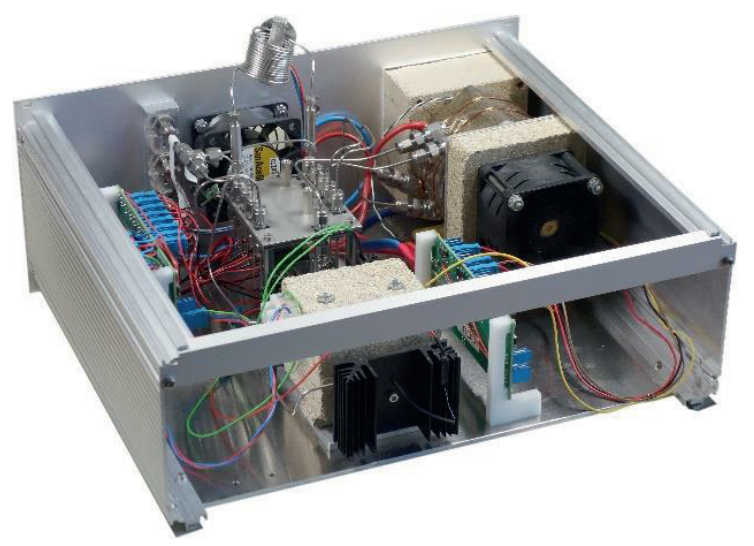

Abb. 12: Prototyp des FES mit Steuerung, Injektion und chromatographischer Trennung, integriert in einem 19Zoll-Gehäuse.

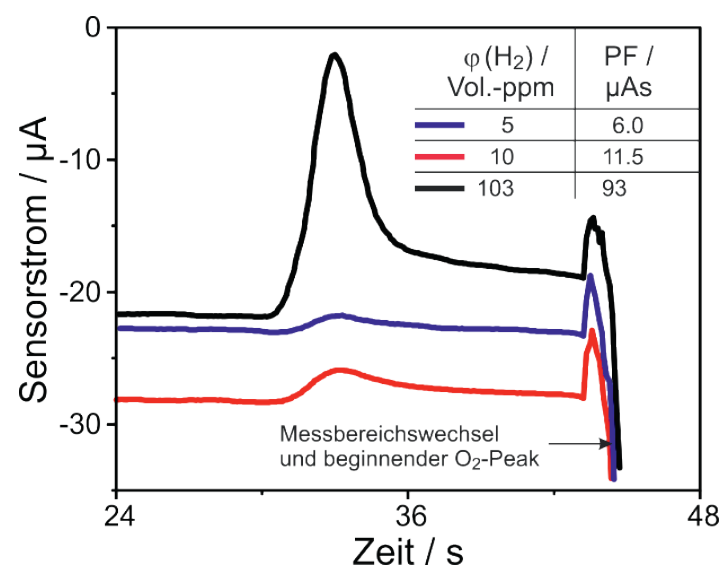

Abb. 13: Ausschnitte aus Chromatogrammen, aufgenommen mit einem coulometrischen Detektor gemäß Abb. 7.

\section{Diskussion}

Die Entwicklung einer modernen Wasserstoffwirtschaft ist untrennbar mit der Schaffung neuer Konzepte für die Gewährleistung der Anwendungssicherheit und der Einsatzqualität verknüpft. Bedingt durch die Besonderheiten von $\mathrm{H}_{2}$ als Energieträger und Grundstoff werden dafür hochselektive Sensoren und automatische Sicherheitsvorrichtungen benötigt. Im Verbundvorhaben „Prozess- und Sicherheitssensorik für das Wasserstoff-Qualitätsmanagement (HyProS)" wurden deshalb neuartige Wasserstoffsensoren für eine zukünftige $\mathrm{H}_{2}$-Infrastruktur zur Herstellung, Verteilung und Applikation von $\mathrm{H}_{2}$ aus erneuerbaren Energiequellen entwickelt [20]. Im Fokus standen fünf verschiedene Sensorprinzipien, deren Einsatzfähigkeit nachgewiesen wurde. Unter Nutzung des Spillover-Effektes, des $\mathrm{H}_{2}$-Nachweises mittels Raman-Streuung, der Kombination verschiedener Einzel-Gassensoren zu einem neuartigen Multisensor, der Wechselwirkung zwischen $\mathrm{H}_{2}$ und Palladium sowie der coulometrischen Umsetzung von $\mathrm{H}_{2}$ konnten für alle Sensorprinzipien erste praxistaugliche Versuchsmuster geschaffen werden. Im Vorhaben durchgeführte Feldtests belegen die Einsatzfähigkeit in modernen Anlagen zur Herstellung, Lagerung und Verarbeitung sowie zum Transport von $\mathrm{H}_{2}$.

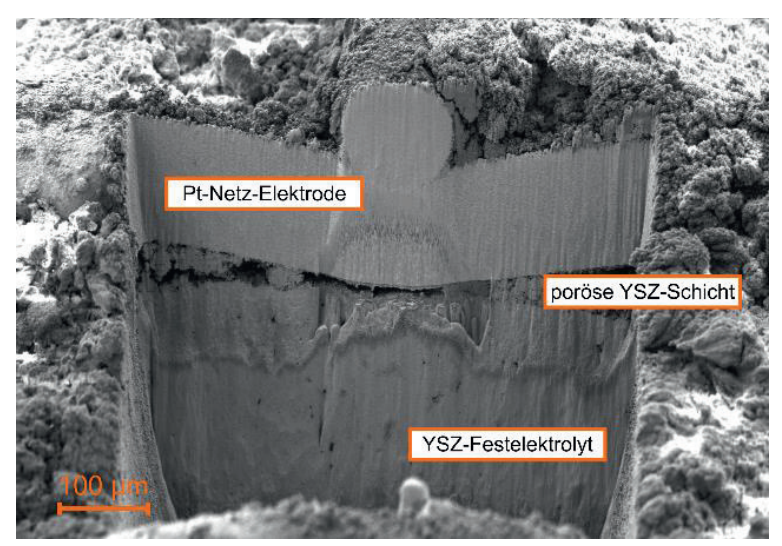

Abb. 14: REM-Aufnahme eines Materialverbundes aus dicht gesintertem YSZ, poröser YSZ-Schicht und PlatinNetzelektrode, Querschliff mittels Laserablation. 


\section{Literatur}

[1] RAMACHANDRAN, R. ; MENON, R.K.: An overview of industrial uses of hydrogen. In: Int $J$ Hydrogen Energ 23 (1998), Nr. 7, S. 593-598; doi: 10.1016/S03603199(97)00112-2

[2] STAFFELL, I. ; SCAMMAN, D. ; VELAZQUEZ ABAD, A. ; BALCOMBE, P. ; DODDS, P.E. ; EKINS, P. ; SHAH, N. ; WARD, K.R.: The role of hydrogen and fuel cells in the global energy system. In: Energ Environ Sci 12 (2019), Nr. 2, S. 463-491; doi: 10.1039/ C8EE01157E

[3] SINIGAGLIA, T. ; LEWISKI, F. ; MARTINS, M.E.S. ; SILUK, J.C.M.: Production, storage, fuel stations of hydrogen and its utilization in automotive applicationsa review. In: Int J Hydrogen Energ 42 (2017), Nr. 39, S. 24597-24611; doi: 10.1016/j.ijhydene.2017.08.063

[4] MAROUFMASHAT, A. ; FOWLER, M.: Transition of Future Energy System Infrastructure; through Powerto-Gas Pathways. In: Energies 10 (2017), Nr. 8, S. 1089-1111; doi: 10.3390/en10081089

[5] FERRERO, D. ; GAMBA, M. K LANZINI, A. ; SANTARELLI, M.: Power-to-Gas Hydrogen: technoeconomic assessment of processes towards a multipurpose energy carrier. In: Energy Procedia 101 (2016), S. 50-57; doi: 10.1016/j.egypro.2016.11.007

[6] KNOSALA, K. ; KOTZUR, L. ; RÖBEN, F.T.C. ; STENZEL, P ; BLUM, L ; ROBINIUS, M. ; STOLTEN, D.: Hybrid Hydrogen Home Storage for Decentralized Energy Autonomy. In: Int J Hydrogen Energ 46 (2021), Nr. 42, S. 21748-21763; doi: 10.1016/j.ijhydene.2021. 04.036

[7] ASTBURY, G.R. ; HAWKSWORTH, S.J.: Spontaneous ignition of hydrogen leaks. In: Proceedings of International Conference on Hydrogen Safety Pisa, Italy, 2005

[8] KESSLER, A. ; SCHREIBER, A. ; WASSMER, C. ; DEIMLING, L. ; KNAPP, S. ; WEISER, V. ; SACHSENHEIMER, K. ; LANGER, G. ; EISENREICH, N.: Ignition of Hydrogen Jet Fires from High Pressure Storage. In: Proceedings of International Conference on Hydrogen Safety Brussels, Belgium, 2013

[9] BUTLER, M.S. ; MORAN, C.W. ; SUNDERLAND, P.B. ; AXELBAUM, R.L.: Limits for hydrogen leaks that can support stable flames. In: Int J Hydrogen Energ 34, (2009), S. 5174-5182, doi: 10.1016/j.ijhydene.2009. 04.012

[10] ISO 26142, ,Hydrogen detector apparatus-stationary applications', 2010.

[11] HÜBERT, T. ; BOON-BRETT, L. ; BLACK, G. ; BANACH, U.: Hydrogen sensors - A review. In: Sensor Actuat B-Chem 157 (2011), Nr. 2, S. 329-352; doi: 10.1016/j.snb.2011.04.070

[12] BUTTNER, W. J. ; POST, M.B. ; BURGESS, R. ; RIVKIN, C.: An overview of hydrogen safety sensors and requirements. In: Int J Hydrogen Energ 36 (2011), Nr. 3, S. 2462-2470; doi: 10.1016/j.ijhydene.2010. 04.176

[13] WIENECKE, M. ; GODENRATH, L. ; ZACHARIAS, B. Sensitivität und Selektivität optischer und neuartiger MEMS-Wasserstoffsensoren. In: Tagungsband der 20. GMA/ITG-Fachtagung Sensoren und Messsysteme Nürnberg, 2019, DOI 10.5162/sensoren2019/3.3.2

[14] SOOD, P. ; ZOSEL, J. ; MERTIG, M. ; OELßNER, W. ; HERRMANN, O. ; WORATZ, M.: Development and test of a highly sensitive and selective hydrogen sensor system. In: J Sens Sens Syst 9 (2020), S. 309-317; https://doi.org/10.5194/jsss-9-309-2020

[15] RÖßNER, F. ; SIMON, U. ; FRANKE, M. ; ROLAND, U.: Verfahren und Sensor zur Bestimmung der Wasserstoffkonzentration in fluiden Medien. Schutzrecht, DE 10011164B4 (2004)

[16] FROSCH, T.: Aktuelle Entwicklungen der RamanGasspektroskopie für die Wasserstoffsensorik und Energieforschung. In: 7. HYPOS-Dialog „Innovative Wasserstoffsensorik" Halle, 19.6.2019.

[17] KIESEWETTER, O. ; KRAUßER, A. ; KIESEWETTER, N. ; MÜLLER, J. ; BOSE, M. ; MAY, M.: Innovative hydrogen sensors in fuel cell vehicles. In: Proceedings of SMSI 2021-Sensor and Measurement Science International Nürnberg, 2021; DOI 10.5162/SMSI2021 /B1.1

[18] GRAFF, A. ; MÜNCHGESANG, W. ; ALTMANN, F. ; HIMCINSCHI, C. ; KÖHLER, T. ; SOOD, P. ; ZOSEL, J. ; MERTIG, M.: Failure analysis of overloaded coulometric hydrogen sensor. In: Proceedings of SMSI 2021-Sensor and Measurement Science International Nürnberg, 2021; DOI 10.5162/SMSI2021/B1.2.

[19] HANF, S. ; KEINER, R. ; YAN, D. ; POPP, J. ; FROSCH, T.: Fiber-Enhanced Raman Multigas Spectroscopy: A Versatile Tool for Environmental Gas Sensing and Breath Analysis. In: Anal Chem 86 (2014); Nr. 11, S. 5278-5285; doi: 10.1021/ac404162w

[20] MERTIG, M ; Kiesewetter, O.: HyProS: Neue Sensorlösungen für die Wasserstoffwirtschaft. In: 6 . HYPOS-Forum, Leipzig, HYPOS e.V., 03.-04.11.2020

\section{Danksagung}

Das diesem Beitrag zugrundeliegende Vorhaben wurde mit Mitteln des Bundesministeriums für Bildung und Forschung unter dem Förderkennzeichen 03ZZ0724 gefördert. Das Kurt-Schwabe-Institut Meinsberg wird mitfinanziert durch Steuermittel auf der Grundlage des vom Sächsischen Landtag beschlossenen Haushaltes. 